\section{Commentary: Exquisite surgical techniques and comprehensive oncologic theory are two fundamental requirements of learning lung cancer surgery}

\author{
Ke-Neng Chen, MD, PhD
}

In 1933, Graham and Singer ${ }^{1}$ successfully performed the first pulmonary resection for lung cancer, opening the practice of lung cancer treatment for humans. Since then, the surgical technique for lung cancer has undergone tremendous changes and progress, and the quality of the surgical technique has been a guarantee of a satisfactory curative effect. Therefore, training of surgical technique has long been the topic of thoracic surgery. ${ }^{2}$ At the same time, the progress of oncology has completely changed the domain of single surgical treatment of lung cancer, which should be regarded as the basic training of thoracic surgery.

\section{BOTH SURGERY AND NEOADJUVANT THERAPY ARE NEEDED FOR LOCALLY ADVANCED LUNG CANCER}

Surgery is the initial approach to treating lung cancer, and substantial evidence shows that surgery is still the most effective treatment for early-stage lung cancer. It is no exaggeration to say that surgical treatment of early-stage lung cancer has a long and efficacious history. However, lung cancer surgery is a "mechanical, cold weapon" for dealing with a complex biological problem, and the curative effect of surgery for advanced lung cancer is extremely limited. From this point of view, surgical treatment for lung cancer, particularly for locally advanced lung cancer, is also a passive and unaided choice. Fortunately, the treatment strategy for lung cancer has developed rapidly with the emergence of radiotherapy and chemotherapy in the 1970s, the availability of

\footnotetext{
From Thoracic Surgery Peking University Cancer Hospital, Beijing, China Disclosures: The author reported no conflicts of interest.

The Journal policy requires editors and reviewers to disclose conflicts of interest and to decline handling or reviewing manuscripts for which they may have a conflict of interest. The editors and reviewers of this article have no conflicts of interest.

Received for publication July 24, 2021; revisions received July 24, 2021; accepted for publication Aug 11, 2021; available ahead of print Aug 16, 2021.

Address for reprints: Ke-Neng Chen, MD, PhD, No. 52, Fucheng Rd, Haidian District, Beijing, China, 100142 (E-mail: chenkeneng@bjmu.edu.cn).

JTCVS Techniques 2021;9:153-4

2666-2507

Copyright (c) 2021 The Author(s). Published by Elsevier Inc. on behalf of The American Association for Thoracic Surgery. This is an open access article under the CC BY-NC-ND license (http://creativecommons.org/licenses/by-nc-nd/4.0/).

https://doi.org/10.1016/j.xjtc.2021.08.017
}
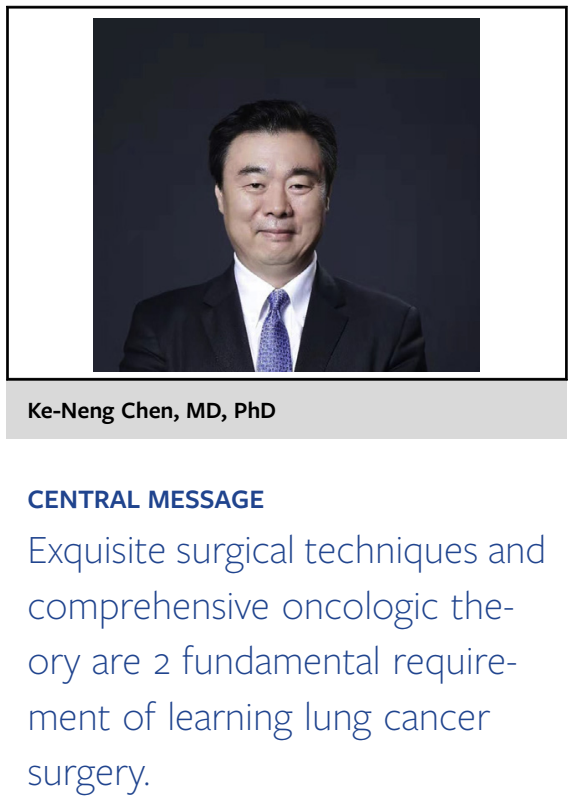

targeting drugs at the beginning of the 21st century, and more recent advances in immunotherapy. Individualized treatment based on systemic therapy has been realized fully, and the curative effect on locally advanced and even advanced lung cancer has been improved greatly. Surgeons must pay attention to this progress and adequately implement procedures to maximize patient survival. It is not difficult to see that the patients with video-assisted thoracoscopic surgery sleeve lobectomy involving bronchus/and pulmonary vessels should have a central type of lung cancer, and most of these patients should have locally advanced disease. Therefore, theoretically, this group of patients particularly needs preoperative systemic induction therapy (in this study, 14 of 127 patients had neoadjuvant chemotherapy, 84 patients had adjuvant chemotherapy, and 6 patients had adjuvant radiotherapy). ${ }^{3}$ Appropriate preoperative induction therapy after sufficient multidisciplinary discussion is critically important for these patients. Induction therapy has the following advantages: (1) the downstaging of tumor can reduce the difficulty of resection and increase the probability of R0 resection; (2) the downstaging of tumor can improve long-term efficacy; (3) the downstaging of tumor may lead to reduction in the extent of resection; and (4) it may possibly eradicate micrometastasis. However, in clinical practice, it is not common for patients with central locally advanced lung cancer to receive preoperative induction therapy (only 14 patients received neoadjuvant chemotherapy in this study). This limitation may affect the therapeutic effect, and insufficient tumor downstaging will lead to unnecessary expanded surgery (surgery performed over a larger area). Therefore, 
neoadjuvant therapy should be an important part of thoracic surgery training and emphasized in publications about surgery (including learning curve).

The success of pneumonectomy in the treatment of lung cancer in 1933 and the principle of lobectomy for lung cancer treatment established by Ginsburg and Rubinstein ${ }^{4}$ have had an important impact on clinical practice, and their basic principles are still in use today. However, more recently, lung cancer is no longer viewed as traditional "lung cancer" but 2 different types of lung cancer: solid non-small cell lung cancer, which has the traditional characteristics, and adenocarcinoma with a ground-glass opacity (GGO) component. For the latter, we must have a new understanding: (1) the new classification of lung adenocarcinoma proposed by Travis and colleagues $^{5}$ introduces "multidisciplinary" evaluation of malignant biological behavior of lung adenocarcinoma to the single pathologic "gold standard" and its significance. (2) The results of JCOG 0201, JCOG 0804, and JCOG0802 showed that sublobectomy may be appropriate for early-stage lung adenocarcinoma with GGO component. ${ }^{6,7}$ The aforementioned factors indicate the following: (1) for early-stage lung cancer with a GGO component, the prognosis, the extent of resection, and the possibility of lymph node metastasis depend on the size of the tumor, the proportion of GGO to solid component, and the location of the tumor (peripheral or central). (2) Most of the pure GGOs with a diameter less than $3 \mathrm{~cm}$ are indolent tumors; we may have a new understanding of whether surgical intervention is needed, the extent of resection (a tendency toward more limited surgical extent), and the scope of lymph node dissection (may hinder smaller range). Besides these factors, the detection of tumor cells in peripheral blood, which is still a hot research topic, may have an impact on surgical decision-making. Because the scope of surgery for early-stage lung cancer may be small, and there is a question of whether surgical intervention is needed for pure GGO-like lung adenocarcinoma with a diameter of less than $3 \mathrm{~cm}$, surgeons should avoid unnecessary major surgery or even any surgery.

\section{THE BALANCE BETWEEN SURGICAL TRAUMA AND SURVIVAL BENEFIT}

The control of surgical trauma has always been the goal of oncologic surgeons, from the traditional large incision thoracotomy in 1933 to current video-assisted thoracoscopic surgery, from the en bloc hilar ligation to anatomical structure ligation, and from the unselected pneumonectomy to precise segmentectomy. However, regardless of type of "minimally invasive" strategy, trauma is always an issue. The basis of surgical treatment for a tumor is to balance the "unlimited trauma" of the tumor with the "limited trauma" of surgery. We can achieve the purpose of surgical treatment only when the trauma caused by surgery is less than the trauma caused by the tumor. In this regard, sleeve lobectomy is based exactly on the premise of achieving the maximal resection of the tumor (disease trauma) with the minimal extent of resection (surgical trauma). However, we must be clear about the following 2 principles: First, sleeve lobectomy is the alternative surgery for pneumonectomy. If $\mathrm{R} 0$ resection cannot be obtained by sleeve lobectomy, then the surgeon should not hesitate to perform pneumonectomy. Second, if before or after induction treatment, R0 resection can be achieved by simple lobectomy, then the surgical procedure should not be expanded to sleeve lobectomy. Besides, it is inappropriate to use sleeve lobectomy for patients with benign diseases who may be cured by simpler thoracoscopic surgery.

\section{EXCEPT TECHNIQUE TRAINING, MULTIDISCIPLINARY TRAINING SHOULD INCLUDE BOTH ONCOLOGICAL MULTIDISCIPLINARY TEAM AND MEDICAL MULTIDISCIPLINARY TEAM}

To date, the development of lung cancer surgery has been inseparable from our understanding of the anatomy and physiology of the important thoracic organs, the pathoanatomy and pathophysiology of the disease, and the development of anesthesia and intensive care unit technologies. Therefore, the training of thoracic surgery should include basic training of anatomy, physiology, pathology, and related disciplines; such training will ensure the safety of surgery. However, in training surgeons, there seems to be a trend to emphasize technical aspects and neglect theoretical factors.

In summary, in the training of lung surgeons, exquisite surgical techniques and comprehensive oncological theory are 2 fundamental factors that cannot be neglected in learning lung cancer surgery.

\section{References}

1. Graham EA, Singer JJ. Successful removal of an entire lung for carcinoma of the bronchus. JAMA. 1984;251:257-60.

2. Schieman C, Seder CW, D'Amico TA, Grondin SC. General thoracic surgical training in North America: contrasting general thoracic surgery residencies in Canada and the United States. J Thorac Cardiovasc Surg. 2018;156:2379-87.

3. Deng J, Jiang L, Li S, Zhang L, Zhong Y, Xie D, et al. The learning curve of videoassisted thoracoscopic sleeve lobectomy in a high-volume pulmonary center. $J$ Thorac Cardiovasc Surg Tech. 2021;9:143-52.

4. Ginsberg RJ, Rubinstein LV. Randomized trial of lobectomy versus limited resection for T1 N0 non-small cell lung cancer. Lung Cancer Study Group. Ann Thorac Surg. 1995;60:615-22.

5. Travis WD, Brambilla E, Noguchi M, Nicholson AG, Geisinger KR, Yatabe Y, et al. International Association for the Study of Lung Cancer/American Thoracic Society/European Respiratory Society international multidisciplinary classification of lung adenocarcinoma. J Thorac Oncol. 2011;6:244-85.

6. Suzuki K, Koike T, Asakawa T, Kusumoto M, Asamura H, Nagai K, et al; Japan Lung Cancer Surgical Study Group (JCOG LCSSG). A prospective radiological study of thin-section computed tomography to predict pathological noninvasiveness in peripheral clinical IA lung cancer (Japan Clinical Oncology Group 0201). J Thorac Oncol. 2011;6:751-6.

7. Suzuki K, Saji H, Aokage K, Watanabe SI, Okada M, Mizusawa J, et al. West Japan Oncology Group, Japan Clinical Oncology Group. Comparison of pulmonary segmentectomy and lobectomy: safety results of a randomized trial. J Thorac Cardiovasc Surg. 2019;158:895-907. 